1 Asymmetric post-translational modifications regulate the intracellular distribution

2

3 Beatriz Cardoso ${ }^{1 \#}$, Ricardo Letra-Vilela ${ }^{1 \#}$, Catarina Silva-Almeida ${ }^{1}$, Ana Maia Rocha ${ }^{1}$,

$4 \quad$ Fernanda Murtinheira ${ }^{1}$, Carmen Rodriguez $^{2}$, Vanesa Martin $^{2}$ and Federico Herrera ${ }^{1 *}$

5

$6 \quad{ }^{1}$ Cell Structure and Dynamics Laboratory, Instituto de Tecnologia Quimica e Biologica

7 (ITQB-NOVA), Universidade Nova de Lisboa, Oeiras, Portugal.

$8{ }^{2}$ Instituto Universitario de Oncología del Principado de Asturias (IUOPA) and

9 Departamento de Morfología y Biología Celular, Facultad de Medicina, c/Julian

10 Claveria, 33006 Oviedo, University of Oviedo, Spain

11 \# Equal contribution

$12 *$ To whom correspondence should be addressed:

13 Federico Herrera, PhD. Cell Structure and Dynamics Laboratory. Instituto de 14 Tecnologia Quimica e Biologica (ITQB-NOVA). Av. da República, Estação 15 Agronómica Nacional, 2781-901 Oeiras, Portugal. Phone: +(351) 21446 9300; E-mail:

16 fherrera@itqb.unl.pt

17 Running title: Asymmetric PTMs regulate latent STAT3 dimer localization

18 Key words: STAT3, bimolecular fluorescence complementation, dimerization, post19 translational modifications, acetylation

20 Abbreviations: BiFC, bimolecular fluorescence complementation; PTMs, post21 translational modifications

22 Contribution: BC is responsible for most microscopy studies, and RV for cloning, mutagenesis and flow cytometry studies, as well as part of microscopy analyses. CSA and AMR are responsible for the first optimization experiments with the BiFC system and LIF experiments, a small part of which are shown in Suppl. Fig. 1. FM contributed to the microscopy, flow cytometry and immunoblotting at different stages of the project. $\mathrm{CR}$ and VM contributed to the analysis, presentation and interpretation of data. FH had the original idea, designed the experiments, arrange the final figures and analyzed the data, coordinated the project and wrote the manuscript.

\title{
31 Abstract
}


32 Signal Transducer and Activator of Transcription 3 (STAT3) is a ubiquitous and 33 pleiotropic transcription factor that plays essential roles in normal development, 34 immunity, response to tissue damage and cancer. We have developed a Venus-STAT3 35 bimolecular fluorescence complementation (BiFC) assay that allows the visualization 36 and study of STAT3 dimerization and protein-protein interactions in living cells. 37 Inactivating mutations on residues susceptible to post-translational modifications 38 (K49R, K140R, K685R, Y705F and S727A) changed significantly the intracellular 39 distribution of unstimulated STAT3 dimers when the dimers were formed by STAT3

40 molecules that carried different mutations. Our results indicate that asymmetric post41 translational modifications on STAT3 dimers could constitute a new level of regulation 42 of STAT3 signaling.

\section{Significance}

45 The Signal Transducer and Activator of Transcription 3 (STAT3) transcription 46 factor plays key roles in development, immunity, cancer or response to stress or 47 damage. All previous studies on STAT3 dimerization work on a homogenous pool of 48 STAT3 molecules, where all STAT3 molecules are equally modified (i.e."symmetric"). 49 However, this is highly unlikely in a complex intracellular environment, as post50 translational modifications do not necessarily occur with complete efficiency or 51 simultaneously. We demonstrate that asymmetric post-translational modifications 52 change the intracellular distribution of STAT3 dimers more strikingly than symmetric 53 ones. This could mean a new level of regulation of STAT3 activity, and therefore a new 54 possible therapeutic target. Our results could be highly relevant for other protein 55 complexes regulated by post-translational modifications beyond STAT3. 


\section{lbodyIntroduction}

The Signal transducer and activator of transcription 3 (STAT3) is a conserved transcription factor that plays key roles in development, immunity, response to injury and cancer $(1,2)$. STAT3 homodimerization, post-translational modification (PTM) and intracellular location are key events in its biological functions. STAT3 is canonically activated by phosphorylation at Y705 upon stimulation with a variety of cytokines and growth factors. However, unstimulated STAT3 also dimerizes, is found in the nucleus, binds to DNA and controls the transcription of a specific set of genes, different from phosphorylated STAT3 (1-5). STAT3 can be also found in the mitochondria, where it is necessary for normal activity of the electron transport chain $(6,7)$. This function is independent of its activity as a transcription factor and Y705 phosphorylation, but dependent on S727 phosphorylation $(1,6,7)$. Other PTMs can regulate the behavior and function of STAT3, such as acetylation at K49 or K685 $(3,8,9)$ or dimethylation at K49 or K140 (10,11). Although dimethylation of the K49 or K140 residues is induced by stimulation with cytokines and is favored by STAT3 phosphorylation, there is basal K49 (but not K140) dimethylation in unstimulated STAT3 (10), and the same happens with STAT3 acetylation $(8,9)$. Literature on STAT3 generally assumes that STAT3 homodimers are formed by two identically modified molecules. However, this is highly unlikely in a complex intracellular context, as PTMs do not occur in all the pool of STAT3 molecules at the same time or with the same efficiency. Here, we aimed at determining the relative contribution of residues K49, K140, K685, Y705 and S727 to the dimerization and intracellular distribution of STAT3 homodimers.

\section{Material and Methods}

We developed a suit of plasmids to study STAT3 homodimerization in living cells, based on bimolecular fluorescence complementation $(\mathrm{BiFC})(12)$. Briefly, the cDNA sequence of STAT3-alpha was fused to the sequence of two complementary, nonfluorescent fragments of the Venus protein (Venus 1, amino acids 1-157; and Venus 2, amino acids 158-238)(Fig. 1A), and inserted in a pcDNA 3.3 TOPO backbone (Invitrogene). When STAT3 dimerizes, the Venus fragments are brought together and reconstitute the fluorophore, the fluorescence being proportional to the amount of dimers (Fig. 1A). Deletion mutants lacking the C-terminus (DelCT) were produced by PCR-mediated subcloning using full-length Venus-STAT3 BiFC constructs as templates. The original lysine $(\mathrm{K})$ residues on positions 49, 140 and 685 were replaced by arginine $(\mathrm{R})$ residues, the tyrosine $(\mathrm{Y})$ residue on position 705 by phenylalanine $(\mathrm{F})$ 
91 and the serine (S) residue on position 727 by alanine (A)(Fig. 1A). Supplementary

92 Table I shows the primers used for cloning and mutagenesis were. Detailed methods for

93 site-directed mutagenesis, cell culture and transfection, fluorescence microscopy, flow

94 cytometry and immunoblotting were described elsewhere (12). All BiFC constructs

95 were deposited in Addgene (https://www.addgene.org/). HeLa human cervix

96 adenocarcinoma cells and HEK293 human embryonic kidney cells were acquired from

97 ATCC (references CRM-CCL-2 and CRL-1573, respectively), Leukemia inhibitory

98 factor (LIF) from R\&D systems (MN, USA), and Stattic from Selleckchem (TX, USA).

\section{Results}

Transfection of HEK293 or HeLa cells with the wild-type (WT) pair of VenusSTAT3 BiFC constructs led to successful expression of the chimeric proteins V1STAT3 and V2-STAT3 (Figs. 1B and 1C, Suppl. Fig. 1). Fluorescence was primarily cytoplasmic in both cell lines, with low but visible nuclear signal (Fig. 1C, Suppl. Fig. 1B). Incubation with Leukemia Inhibitory Factor (LIF, $100 \mathrm{ng} / \mathrm{ml}$ ) induced STAT3 phosphorylation and translocation to the nucleus in HEK293 and HeLa cells (Suppl.

107 Fig. 1B-C), but it did not enhance STAT3 dimerization (Fig. 1B, Suppl. Fig. 1D). 108 Incubation with STAT3 inhibitor Stattic $(5 \mu \mathrm{M})$ or removal of the C-terminus 109 containing the SH2 domain partially prevented STAT3 dimerization (Fig. 1B), 110 consistent with previous reports $(13,14)$. On the other hand, single or double 111 Y705F/S727A phosphoresistant mutants did not decrease fluorescence (Fig. 1B). These 112 results support relevant evidence indicating that STAT3 dimerization is actually 113 independent of phosphorylation $(1,5,14)$. The behavior of the Venus-STAT3 BiFC 114 system is therefore consistent with previous reports for tagged STAT3.

115 In order to analyze the intracellular location of unstimulated STAT3 homodimers, 116 we classified cells qualitatively in three categories that are mutually exclusive (their 117 sum is $100 \%$ of cells), according to the relative intensity and location of the 118 fluorescence signal (Fig. 1C-1D): 1) predominantly in the cytoplasm (e.g. WT pair), 2) 119 predominantly in the nucleus (e.g. upon LIF induction, Suppl. Fig. 1B), or 3) 120 homogeneously distributed through nucleus and cytoplasm (e.g. Y705F pair). We also 121 determined the percentage of cells with mitochondrial signal or intracellular inclusions 122 (Suppl. Fig. 2). Although changes in patterns of STAT3 dimer distribution were 123 observed in several symmetric BiFC pairs, only Y705F and S727A pairs induced 
124 significant increases in the percentage of cells with homogeneous nucleocytoplasmic

125 fluorescence or inclusions, respectively (Fig. 1D).

126 We made use of the unique properties of our BiFC system to determine the relative

127 contribution of each residue to the dimerization and intracellular distribution of

128 unstimulated STAT3 dimers. We combined all possible inactivating PTM mutations

129 with each other, but no combination had a consistent effect on STAT3 dimerization as

130 determined by flow cytometry (Suppl. Fig. 3). However, the intracellular distribution of

131 STAT3 homodimers was significantly altered by specific combinations of STAT3

132 molecules (Fig. 2A). Unlike the K49R symmetric pair, K49R asymmetric combinations

133 dominantly induced an increase in cells with homogeneous nucleocytoplasmic

134 fluorescence at the expense of cytoplasmic location (Fig. 2A), similar to the Y705F

135 symmetric pair. K140R- or K685R-containing pairs showed some tendency to shift

136 cytoplasmic location towards nucleus, but only the K140R+S727A combination

137 achieved significance. This phenotype was almost identical to the Y705F+S727A

138 asymmetric pair (Fig. 2A).

139 We then pooled and analyzed all results according to the number and type of PTM

140 mutations present in the each BiFC pair. Combinations carrying any one (asymmetric)

141 or two K-R substitutions (symmetric or asymmetric) significantly increased

142 mitochondrial translocation, while decreasing the percentage of cells with STAT3

143 dimers predominantly in the cytoplasm (Fig. 2B). Asymmetric combinations of one K-R

144 substitution and one phosphoresistant mutant also increased nuclear translocation, but

145 only 2xK-R combinations increased homogeneous nucleocytoplasmic distribution.

146 Combinations carrying any two phosphoresistant mutations (symmetric or asymmetric)

147 had no significant effect on cellular distribution of STAT3 homodimers (Fig. 2B).

148 These results indicate that specific asymmetric PTMs on STAT3 dimers can prevent

149 their nuclear import/export. This was later confirmed by pooling the data according to

150 whether the STAT3 pair was symmetric or asymmetric in their PTM mutations (Fig.

$1512 \mathrm{C})$. We found that only asymmetric PTM mutant combinations increased

152 nucleocytoplasmic or nuclear distribution at the expense of decreasing cytoplasmic

153 localization of STAT3 homodimers. Asymmetric combinations were also sufficient to

154 produce an increase in mitochondrial localization of STAT3 dimers (Fig. 1C).

\section{Discussion}


156 Our results indicate that asymmetric PTMs could constitute a new level of 157 regulation of unstimulated STAT3 behavior and function. Most STAT3 molecules are 158 not phosphorylated in the absence of extracellular stimuli, and this proportion is 159 reversed shortly after cytokines bind to their corresponding membrane receptors (Suppl.

160 Fig. 1C). However, cells often show small amounts of phosphorylated STAT3 in resting 161 state (Suppl. Fig. 1C) and, conversely, cytokine-stimulated STAT3 induces the de novo 162 transcription of new STAT3 molecules that are not necessarily phosphorylated $(1,2)$. 163 This indicates that unphosphorylated and phosphorylated STAT3 should coexist at 164 similar levels in many situations, and the literature presents evidence that this could be 165 equally true for other STAT3 PTMs induced by cytokines (8-10).

166 To the best of our knowledge, there is no direct empirical evidence in the literature 167 showing that asymmetric STAT3 dimers actually happen in living cells, and such 168 demonstration would be currently extremely difficult from a technical point of view, 169 even in vitro. Previous studies most often rely on systems that do not differentiate 170 between monomers and dimers (2,3,6-11), and/or that produce a single population of 171 STAT3 molecules, either mutated or normal $(5,14,15)$. And yet, in the crowded and 172 diverse intracellular environment, the probability for two identical STAT3 molecules to 173 form a dimer (or for a dimer to be modified in both molecules simultaneously and in the 174 same residues) should be low, although it could certainly be enhanced by either total 175 absence or presence of stimuli. Our results point in this exciting direction, and open a 176 series of interesting questions. If asymmetric STAT3 dimers actually happen, do they 177 regulate specific sets of genes? Do they enable gradation of STAT3 transcriptional or 178 mitochondrial activities? And if they do not happen, how do cells manage to achieve 179 perfectly symmetrical STAT3 dimers with such high efficiency? Given the essential 180 roles of STAT3 in development, immunity, tissue stress and cancer, addressing these 181 questions could have important implications for the diagnosis, treatment and 182 understanding of a wide spectrum of human pathologies.

\section{Acknowledgements}

184 The authors thank the Advanced Imaging Unit from Gulbenkian Science Institute 185 and Dr. Sixto Herrera for support with bioimaging and flow cytometry, and statistics, 186 respectively. FH was supported by Project LISBOA-01-0145-FEDER-007660 (Cellular 187 Structural and Molecular Microbiology) funded by FEDER funds through 188 COMPETE2020 - Programa Operacional Competitividade e Internacionalização (POCI) 
189 and by national funds through Fundação para a Ciência e Tecnologia (FCT, Ref.

190 IF/00094/2013/CP1173/CT0005 and PTDC/MED-NEU/31417/2017). RLV and FM

191 were supported by fellowships from FCT (Refs. PD/BD/128163/2016 and

192 SFRH/BD/133220/2017, respectively).

193

194

\section{References}

195

1. Srivastava J, DiGiovanni J. Non-canonical Stat3 signaling in cancer. Mol Carcinog. 2016 Dec;55(12):1889-98.

2. Yang J, Chatterjee-Kishore M, Staugaitis SM, Nguyen H, Schlessinger K, Levy DE, et al. Novel roles of unphosphorylated STAT3 in oncogenesis and transcriptional regulation. Cancer Res. 2005 Feb 1;65(3):939-47.

6. Wegrzyn J, Potla R, Chwae YJ, Sepuri NBV, Zhang Q, Koeck T, et al. Function of

3. Dasgupta M, Unal H, Willard B, Yang J, Karnik SS, Stark GR. Critical Role for

7. Zhang Q, Raje V, Yakovlev VA, Yacoub A, Szczepanek K, Meier J, et al. nonactivated STAT3 molecules demonstrated in living cells using bioluminescence resonance energy transfer $\square$ : a new model of STAT activation? J Leukoc Biol. 2004 May;75(5):792-7.

9. Ray S, Boldogh I, Brasier AR. STAT3 NH2-terminal acetylation is activated by the

8. Kang HJ, Yi YW, Hou S-J, Kim HJ, Kong Y, Bae I, et al. Disruption of STAT3Mitochondrial localized Stat3 promotes breast cancer growth via phosphorylation hepatic acute-phase response and required for IL-6 induction of angiotensinogen. Gastroenterology. 2005 Nov;129(5):1616-32.

10. Dasgupta M, Dermawan JKT, Willard B, Stark GR. STAT3-driven transcription depends upon the dimethylation of K49 by EZH2. Proc Natl Acad Sci. 2015 
Mar;112(13):3985-90.

11. Yang J, Huang J, Dasgupta M, Sears N, Miyagi M, Wang B, et al. Reversible methylation of promoter-bound STAT3 by histone-modifying enzymes. Proc Natl Acad Sci U S A. 2010 Dec 14;107(50):21499-504.

12. Branco-Santos J, Herrera F, Poças GM, Pires-Afonso Y, Giorgini F, Domingos PM, et al. Protein phosphatase 1 regulates huntingtin exon 1 aggregation and toxicity. Hum Mol Genet. 2017 Oct 1;26(19):3763-3775. of STAT3 Activation and Dimerization. Chem Biol. 2006 Nov;13(11):1235-42. Muller-Newen G. Consequences of the disease-related L78R mutation for dimerization and activity of STAT3. J Cell Sci. 2014 May 1;127(Pt 9):1899-910. quantification of dynamic STAT3 homodimerization in living cells using homoFluoppi. Sci Rep. 2018 Feb 5;8(1):2385.

\section{Figure Legends}

Figure 1. A Venus-STAT3 BiFC system allows the visualization and study of acids 1-158 (Venus 1, V1) and 159-238 (Venus 2, V2) were fused to the N-terminus of the STAT3 sequence in two independent constructs. K49, K140, K685, Y705 and S727 residues can be post-translationally modified, and were inactivated in both V1- and V2STAT3 constructs by site-directed mutagenesis. B, Wild-type Venus-STAT3 constructs produced fluorescence in HeLa cells, and it was monitored by flow cytometry 24 hours after transfection with BiFC constructs. Incubation with Leukemia Inhibitory Factor $(100 \mathrm{ng} / \mathrm{ml})$ for 2 hours in the absence of serum or the presence of the indicated drugs or mutant BiFC pairs $(\mathrm{n}=3, \mathrm{p}<0.05)$. Results were normalized versus the Wild-type STAT3 pair $(100 \%)$. C, Microscopy pictures of representative cell phenotypes in the different symmetric combinations of BiFC Venus-STAT3 constructs (Incl, inclusions; Scale bar, $20 \mu \mathrm{m})$. D, Percentage of cells displaying fluorescence predominantly in the Nucleus (black bar), predominantly in the Cytosol (white bar), homogeneously distributed in cytoplasm and nucleus (nucleocytoplasmic, grey bar), in the mitochondria or in nonmitochondrial inclusions. Data are shown as the average \pm SEM of $n=12$ (Wild-type, WT) or $n=3$ (rest of combinations) independent experiments. Statistical analysis was carried out by means of a One-way ANOVA followed by a Bonferroni test adjusted for multiple comparisons. *, sign. vs the symmetric Wild-type STAT3 pair, p<0.05.

263

Figure 2. Asymmetric STAT3 PTMs regulate intracellular distribution of STAT3 homodimers. A, Intracellular distribution of fluorescence in asymmetric combinations of Venus-STAT3 BiFC constructs (and the WT symmetric pair as reference). Data are shown as the average of $n=12$ (Wild-type, WT) or $n=3$ (rest of combinations) 
264 independent experiments \pm SEM. Statistical analysis was carried out by means of a 265 One-way ANOVA followed by a Bonferroni test adjusted for multiple comparisons. *, 266 significant vs the symmetric Wild-type STAT3 pair, *, p<0.05, ** p<0.01. B-C, The 267 same original data, but pooled according to the number and nature of substitutions (B) 268 or the symmetry or asymmetry of substitutions (C) in the STAT3 homodimer, and 269 represented as box plots. The limits of the boxes represent the smallest and largest 270 values, the straight line represents the median, the dashed line represents the average, 271 and the dotted line represents the average for wild-type STAT3 pair. Statistical analysis was carried out on the Average \pm SEM of each pool of data $(1 \times Y F / S A: 1 x K R, n=6$; $2 \mathrm{xYF} / \mathrm{SA}, \mathrm{n}=3 ; 2 \mathrm{xKR}, \mathrm{n}=6$; sym, $\mathrm{n}=5 ;$ asym, $\mathrm{n}=10$ ). Statistical analysis was carried out by means of a One-way ANOVA followed by a Bonferroni test adjusted for multiple comparisons. *, significant vs the symmetric Wild-type STAT3 pair, *, p<0.05, ** $\mathrm{p}<0.01$; \#, significant vs $2 \mathrm{xYF} / \mathrm{SA}$ substitution (in B) or the symmetric mutant pairs (in C), \#, p<0.05, \#\#, p<0.01. 
A $1207 \quad$ Nucleus $\square$ Cytoplasm $\square$ Nucleocytoplasmic

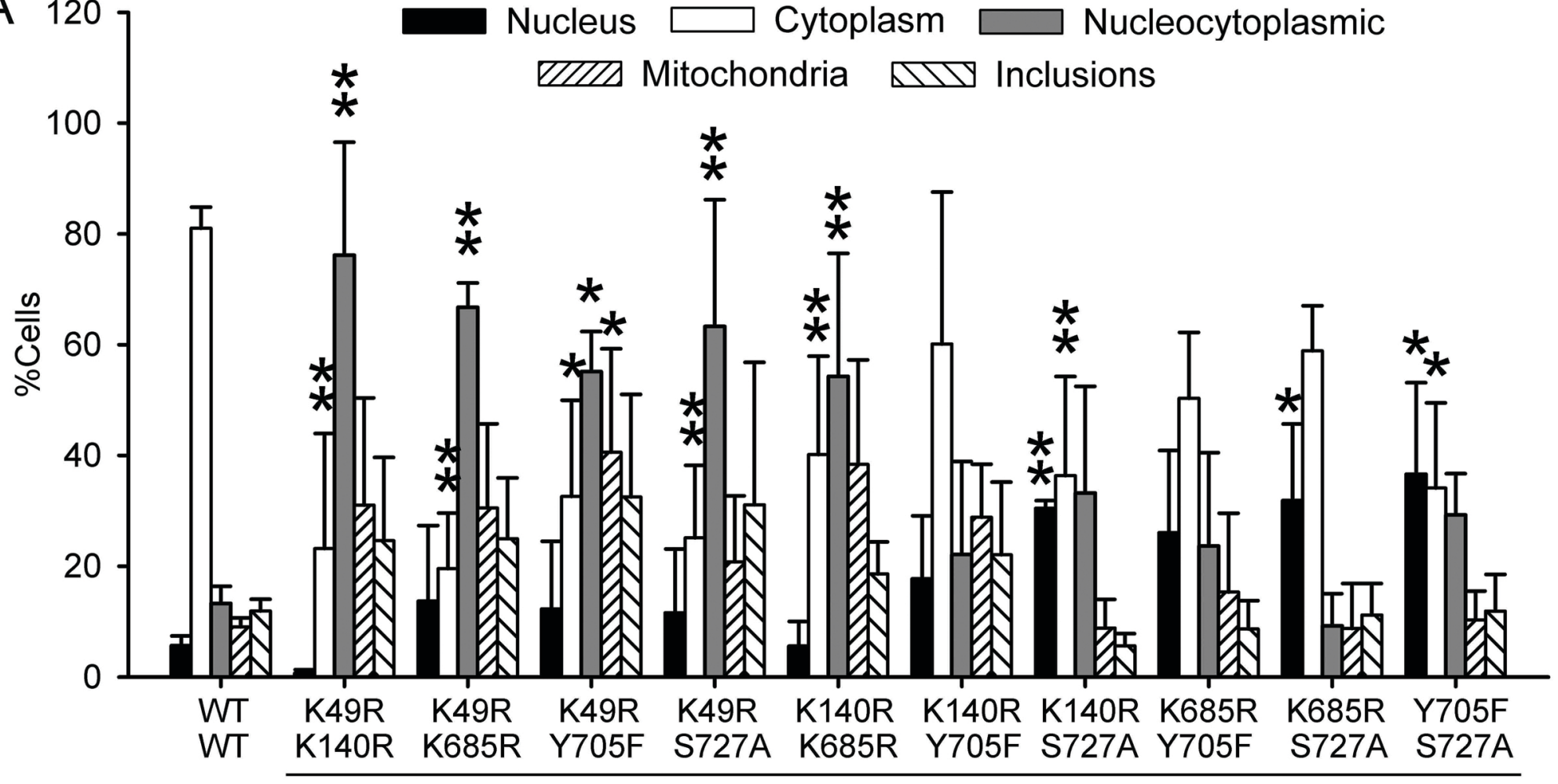

Asymmetric STAT3 dimer combinations

B
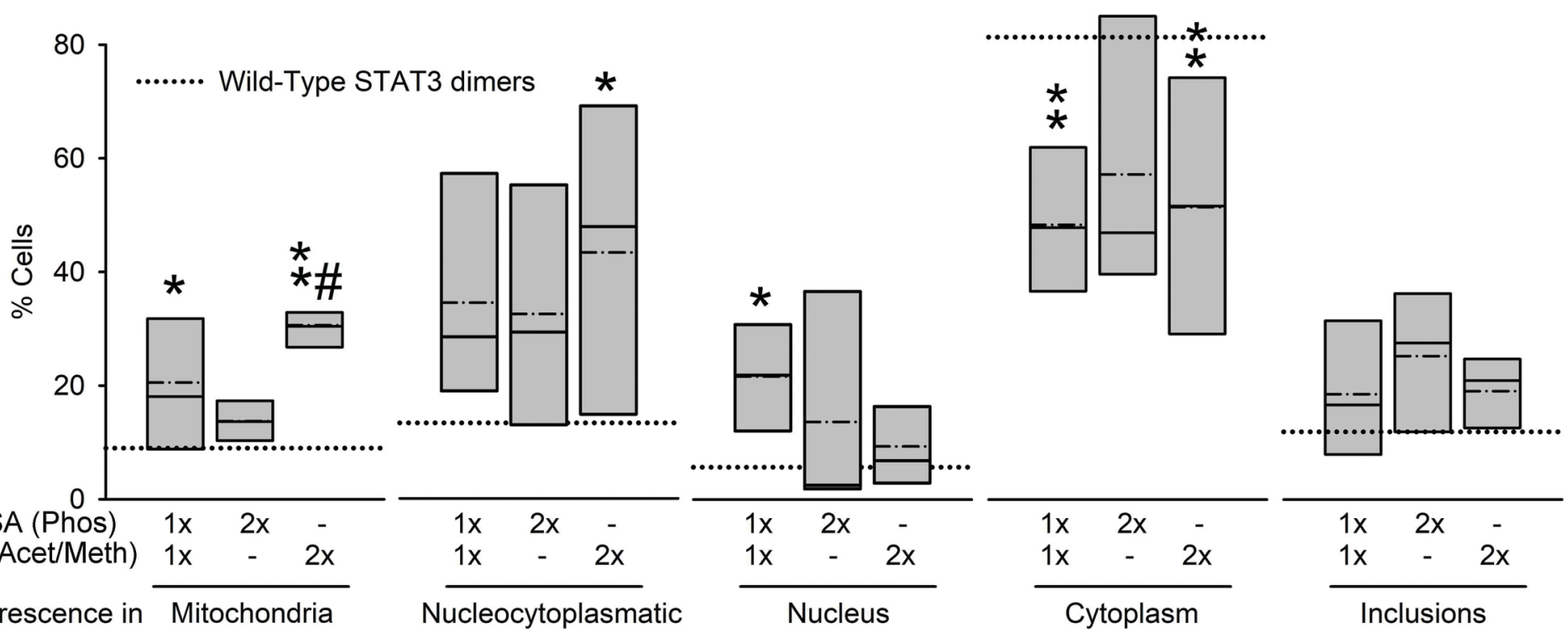
Fluorescence in Mitochondria

C
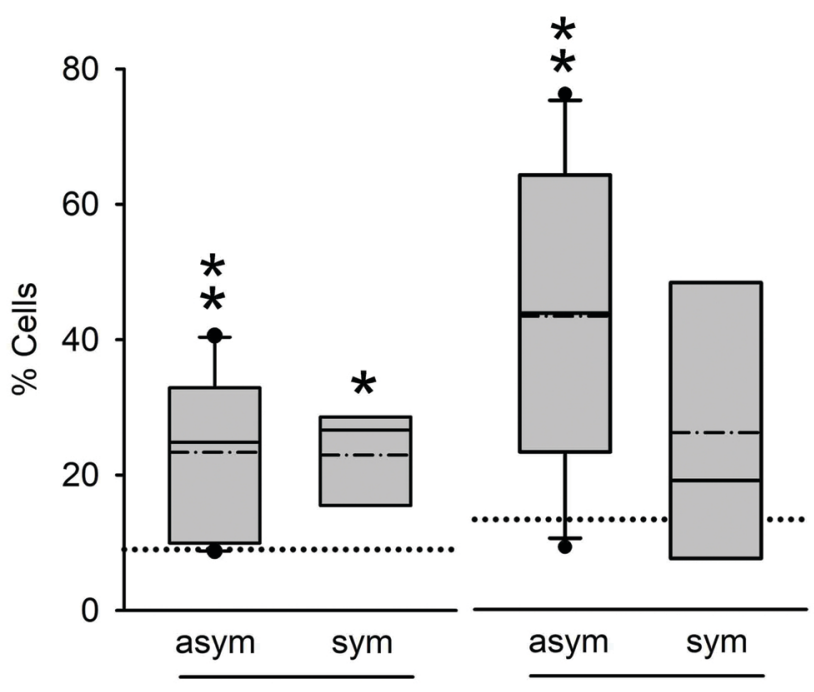

Fluorescence in

Mitochondria

Nucleocytoplasmatic

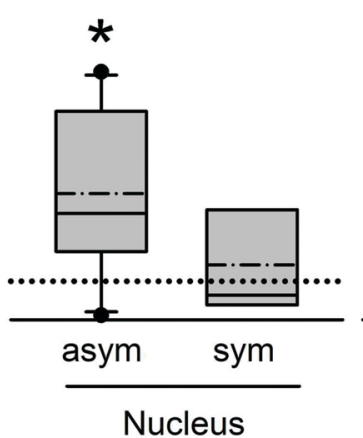

Nucleus
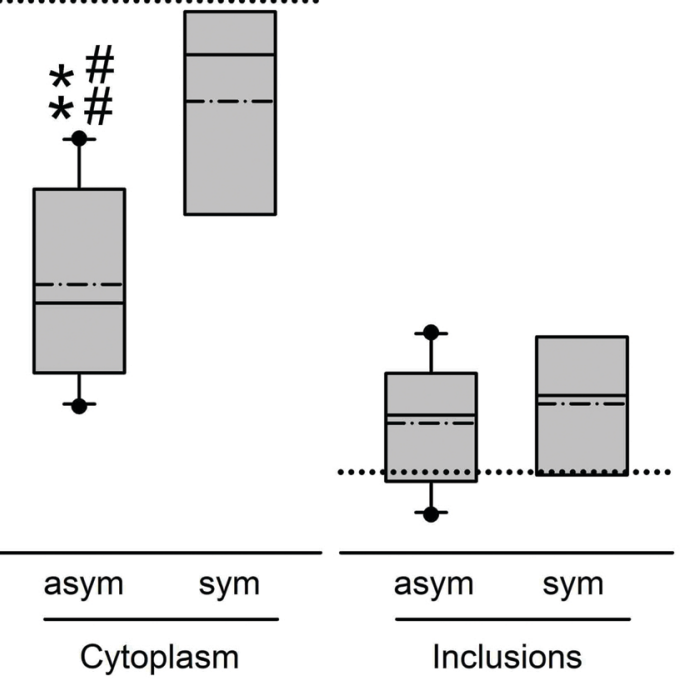